\title{
Preface: Optimization for groundwater characterization and management
}

\author{
Bithin Datta · George Kourakos
}

Keywords Source characterization - Groundwater management - Coastal aquifers - Groundwater contamination - Optimization

\section{Introduction}

Anthropogenic activities conducted in an unplanned manner, and often without a regional-scale view, have led to over-exploitation and wide spread deterioration of groundwater quality. In order to address all the associated groundwater management issues on a larger regional scale, it is necessary to (1) obtain adequate and reliable information regarding the complex geology of the aquifer; (2) develop suitable mathematical simulation models incorporating reliable estimates of hydrogeologic and geochemical parameters, to describe the likely impacts of human interventions; and (3) utilize a suitably formulated decision model capable of prescribing as a solution a set of strategies for optimal management of aquifers, or capable of optimally solving the inverse problem with unknown hydrogeologic or geochemical characteristics. These components are often interrelated. This theme issue, Optimization for Groundwater Characterization and Management, provides a glimpse of the present state of the art, and points towards some of the future prospects in this area.

Received: 6 July 2015 / Accepted: 10 July 2015

Published online: 30 July 2015

(C) Springer-Verlag Berlin Heidelberg 2015

Published in the theme issue "Optimization for Groundwater Characterization and Management"

\section{B. Datta $(\square)$}

Discipline of Civil Engineering, College of Science Technology and Engineering and Centre for Tropical Water and Aquatic Ecosystem Research, James Cook University, Townsville, QLD 4811, Australia e-mail: bithin.datta@jcu.edu.au

B. Datta

CRC for Contaminant Assessment and Remediation of the Environment (CARE), Mawson Lakes, SA 5095, Australia

G. Kourakos

Department of Land Air and Water Resources, University of California Davis, Davis, CA 95616, USA
Water as a renewable economic resource is expected to be the main focus in the coming decades and groundwater will remain an important component of it. Also, during the last two decades, increasing attention has been focused on the quality and sustainability aspects. It is now well recognized that groundwater availability and its associated quality are equally important. Groundwater will remain an important economic resource. Large-scale management of aquifers for efficient sustainable utilization will require development and application of methodologies and tools for better analysis, prediction of groundwater availability (capable of prescribing strategies for its long-term sustainable use in conjunction with surface water), identification of the pathways and sources of contamination, design of efficient management and monitoring strategies, and efficient design of contamination remediation strategies.

From a management perspective, simulation of the physical processes in an aquifer can provide answers to various 'what if' scenarios. No doubt many sophisticated numerical simulation models have been developed and used as descriptive tools that can answer 'what if' questions. Simulation models generally play descriptive roles as they are able to predict the impact of potential management or remediation strategies. However, use of simulation tools alone ideally requires exhaustive "brute force" searches for the best possible solution or option for a given management problem, often leading to suboptimal solutions due to inadequacies in the search methods; thus, a systematic approach is needed. To this end, systematic approaches in the form of optimization methods have been developed that combine with the simulation models to identify optimal solutions or strategies. These optimization approaches solve an appropriately formulated optimization problem with defined objectives and managerial and/or physical constraints. Examples of some of the earliest attempts to develop a combined simulation and nonlinear optimization method to design reliable and efficient optimal aquifer remedial strategies were presented by Gorelick and Remson (1982) and Gorelick et al. (1984).

Where are we today with respect to practical use of optimization methodology in water resources management? The current situation is such that some important 
issues are still faced by scientists and engineers to make simulation models effective descriptive representations of hydrogeologic systems. These include: What is the best way to incorporate errors and uncertainties in available information regarding the physical system? How can mathematical models be best calibrated to truly represent the hydrologic and hydrogeologic system and processes in a study area? How can efficient schemes of monitoring be determined to help obtain reliable estimates of system parameters? How can the ubiquitous heterogeneity in natural systems be dealt with? Important related issues concern effectively working with the computational complexity of adequate and reliable simulation models representing the physical processes, and improving computational tools to deal with highly nonlinear large-scale systems.

The introduction of an optimization approach (in addition to the preceding) adds some difficulties, however, from a different point of view, in striving to directly reach an optimal solution with respect to the formally stated objective and imposed system constraints. Optimization approaches have distinct advantages. By nature, simulation-optimization methods have prescriptive characteristics that provide optimal solutions in contrast with the descriptive nature of simulation models. However, the true "optimality" of the prescribed solutions is dependent on the assumptions incorporated within the prescriptive models. Sometimes the limitations of the optimization algorithms compel simplification of the system description within the optimal decision models. The application of optimization-based methodologies for management of hydrogeologic systems and groundwater parameter estimation has become widespread during the last two decades. Datta et al. (1989) proposed a unique combination of optimal statistical pattern recognition techniques, optimization-based screening models, and a rule-based expert system for identification of unknown groundwater contaminant sources under uncertainties. Later developments, apart from a purely statistical approach, concentrated on use of linked simulation-optimization methods or surrogate models for optimal characterization of unknown contaminant sources in groundwater systems.

Doherty and Hunt (2010) discussed a number of optimization methods available for solving the parameter identification problem in groundwater systems. In addition, optimization methods provide a tool to evolve strategies for economic management of groundwater resources in conjunction with surface-water use. Also, optimization-based prescriptive models, for example, are capable of delineating the most efficient and economic large-scale strategies to control or remediate contamination of aquifers, and to control saltwater intrusion in coastal aquifers through a planned pumping strategy. One area of focus in the last few years had been the capability of estimating the sources of groundwater pollution using a limited amount of contamination field data so that effective remediation strategies may be formulated. The discussion in Wagner (1995) provides a comprehensive review of some of basic issues and the past developments related to the use of optimization methodologies in the management of hydrogeologic systems, particularly associated with quantity management. The first article in this theme issue of Hydrogeology Journal provides a useful present-day summary of developed optimization models applied to hydrogeologic systems. Initial attempts to develop and apply optimization-based tools to groundwater management are described in Gorelick (1983). Most of these aspects related to development and application of optimization tools in prescriptive decision models are covered by the authors in this theme issue. The last few years have seen heightened research activity in solving the non-unique and often ill-posed problem of identifying unknown sources of groundwater contamination based on sparse field data. This particular topic and the related issue of designing optimal monitoring networks to improve the efficiency of characterizing unknown sources of contamination in terms of location, magnitude and release history are also adequately covered in this theme issue. However, the topics covered in this issue are in no way exhaustive, and only provide a sample of the extensive range of possible application of optimization models and tools in comprehensive characterization and management of hydrogeologic systems.

Some of the earliest attempts to introduce optimization tools and models for management of hydrogeologic systems, i.e., aquifers (Gorelick 1983) were based on linear programming models due to the computational issues related to solution of large-scale nonlinear programming problems at that time. Wagner and Gorelick (1989) integrated statistical techniques and reliability concepts along with nonlinear optimization to develop a two-step method for determining reliable aquifer remediation strategies. Possibly with the exception of optimal monitoring-network-design methods, most realistic applications of optimization methods to modeling, characterizing, or managing the hydrogeologic system require integrated simulation of the flow and/or transport processes within the optimal decision models. This integrated simulation-optimization within the formulated model can ensure acceptable and feasible solutions of the optimization-based models. Therefore, the earliest attempts to incorporate the process simulation within an optimization model mostly limited by the requirement of utilizing linear programming solutions, were based on approximating the hydrogeologic system response as linear responses that lead to the use of the response matrix approach and to some extent lead to the use of influence coefficients (Gorelick et al. 1983; Illangasekare and Morel-Seytoux 1982; Datta and Peralta 1986; McPhee and Yeh 2004). However, the limitations in assuming the validity of linear superposition principles in generally nonlinear hydrogeologic systems lead to the development of other approaches for development of optimal decision models. One of these methods is the embedded optimization technique, where the governing equations representing the physical processes are embedded as binding constraints of the optimization model. As pointed out by Das and Datta (1999), embedded optimization 
models suffer from issues of computational feasibility, especially for large hydrogeologic systems. Therefore, a search for a more efficient computational approach towards developing groundwater quantity and quality models led to the more recent versions of the linked simulation optimization models for large-scale and longterm optimal management and remediation of contaminated aquifers. As noted in Wagner (1995), "The combined simulation and optimization model accounts for the complex behavior of the groundwater system and identifies the best management strategy under consideration of the management objectives and constraints". Some of the relevant issues are also discussed in Aral (2011).

Prior to the wide acceptance of the evolutionary optimization techniques such as genetic algorithm (GA) and simulated annealing (SA), etc., it was observed that external linking of the simulation model was computationally complex when classical nonlinear optimization algorithms were used to solve optimization models for contamination source identification and parameter estimation (Datta et al. 2009, 2011). In this approach, the groundwater flow and transport simulator is linked to the nonlinear optimization model as an external module. The essential links between the simulator and the optimization method are the derivatives or gradient information required to construct the Jacobean matrix for the optimization algorithm. Some of these limitations of using classical optimization algorithms could be overcome using the more direct search procedures, i.e., the evolutionary algorithms such as GA and adaptive SA (ASA), with other more-advanced variants now becoming available. Wagner (1995) discusses the historical perspective and the future prospects of using the linked simulation-optimization approach in various types of groundwater management models, as well as source identification and parameter estimation aspects. However, as noted in Datta et al. (2011), the dimensionality of the optimal search problem increases exponentially with the increase in number of decision variables. The number of iterations between the optimization algorithm and the externally linked simulation models required to obtain an optimal or near optimal solution also increases exponentially as the number of decision variables increases. This was one of the motivations for development and potential application of surrogate models that are trained and validated by repeated use of numerical flow and contaminant transport simulation models (Bhattacharjya and Datta 2005; Kourakos and Mantoglou 2009). These surrogate models then replace the complex numerical simulation models, and perform the role of an approximate simulator. The surrogate models can be based on artificial neural networks (ANN), genetic programming (GP) models, and selforganizing maps (SOM) etc. More recently, the emphasis on computational efficiency has also focused on utilizing parallel processing capabilities especially to solve largescale optimization models for groundwater management (Mirghani et al. 2009). Often a typical linked simulationoptimization model could take days of CPU time to solve a single optimization problem, and the computational

Hydrogeology Journal (2015) 23: 1043-1049 burden can become prohibitive especially when multiple conflicting objectives of aquifer management are considered (Dhar and Datta 2009). Surrogate models or meta models as approximators of physical processes in a coastal aquifer, for example, could be essential to solve a largescale multi-objective management model incorporating highly nonlinear density-dependent coastal processes.

Some of the afore-mentioned difficulties have been satisfactorily addressed and some of the challenges remain. Contributions to this theme issue address most of these major aspects. The main problems that have attracted the majority of attention for development and application of optimization tools and techniques are, for the purposes of this theme issue, subdivided into three groups: (1) parameter and contaminant source characterization and model complexity, (2) coastal aquifer management (seawater intrusion management), and (3) general groundwater management (development of strategies for optimal management of groundwater and surface water including economics, conjunctive use etc.). The articles within these groups are introduced briefly in the following.

\section{Parameter and source characterization and model complexity}

As the first article in this theme issue, Yeh provides a general overview of the various optimization methods that have been applied for solving the inverse problem (Yeh 1986) of parameter identification (estimation), experimental design, and groundwater planning and management. The optimization tools such as, mathematical programming techniques, linear programming, quadratic programming, dynamic programming, stochastic programming, nonlinear programming along with newer evolutionary global optimization methods, i.e., genetic algorithm, tabu search, and simulated annealing, are mentioned. A typical illustrative problem is used to demonstrate the basic optimization model formulation and the solution algorithm. One critical issue faced by practitioners in groundwater management is the criteria for selection of an appropriate model for description of the physical system. The relevant criteria are discussed. The inverse problem for optimal parameter identification is described, including sampling strategies for optimal determination of model parameters. Typical objectives of conjunctive groundwater and surface-water use to meet demands are described. It is, however, clearly stated that the emphasis of the review is very much on groundwater flow problems and not on contaminant transport problems.

Timani and Peralta address an important issue related to an acceptable model of the hydrogeologic processes to be included as a component of the optimal management model for strategy development. It is argued that failure to agree on the validity of a single simulation model often prevents the adoption of consensus management strategies; therefore, a multi-conceptual model optimization (MCMO) can help stakeholders reach a compromise 
strategy. MCMO computes mathematically optimal strategies that simultaneously satisfy analogous constraints and bounds in multiple numerical models that differ in boundary conditions, hydrogeologic stratigraphy, and discretization. Shadow prices and trade-offs guide the process of refining the first MCMO-developed multimodel strategy into a realistic compromise management strategy. The practical application of the MCMO is appropriate for linear and nonlinear aquifer systems. A reconnaissance study demonstrating the MCMO application to the multi-layer Cache Valley (Utah and Idaho, USA) river-aquifer system is presented. A typical groundwater-quantity-management objective that maximizes additional safe pumping (beyond current pumping), subject to constraints on groundwater head and seepage from the aquifer to surface waters, is adopted.

Characterization of unknown groundwater pollution sources still remains a challenging task, especially when very sparse and inadequate initial contaminant concentration measurements are available even for preliminary determination of potential locations, magnitudes and time history of contaminant source activities. Often the inverse problem is difficult to solve as the inverse problem is illposed and the solutions may be non-unique.

Prakash and Datta present and demonstrate an important application of an optimization-based methodology for characterization of unknown pollution sources in contaminated aquifers, integrating sequential and iterative design of optimal monitoring networks. The integrated design and implementation of the monitoring network can sequentially improve the source identification process, and gradually reduce the non-unique and ill-posed nature of the inverse problem by adding new concentration measurements collected as part of a designed monitoring plan. The SA optimization algorithm is used to solve the models for optimal source identification and the monitoringnetwork-design optimization. This sequence is repeated a few times to improve the accuracy of source characterization. The performance of this methodology is evaluated by application to a contaminated aquifer site in New South Wales, Australia, where source location, source-activity initiation time and source-flux (mass per unit time) release history are considered as unknown variables. The efficiency of the proposed methodology is demonstrated for the illustrative contaminated aquifer site.

Gurarslan and Karahan developed an improved model for groundwater-pollution-source identification. In the developed model, the numerical simulations of flow and pollutant transport in groundwater were carried out using external numerical simulation models: MODFLOW and MT3DMS software. The optimization processes were carried out using a differential evolutionary algorithm. The performance of the developed model was tested on two hypothetical aquifer models using both real and noisy observation data. In the first model, the release histories of the pollution sources were determined. The results obtained by the developed model were found to be better than those obtained using existing models for source identification.
Starn et al. address another aspect of optimal hydrogeologic modeling accuracy related to utilization of more measurement data and increasing the complexity or the degree of details in the models used for calibration. It appears it is difficult to establish a definite trade-off between 'optimality' of the simulation models in terms of data utilization and its complexity. Optimal choice of the simulation model is no doubt an important factor in formulating reliable and effective optimal management strategies. The relative merits of model complexity and types of observations employed in model calibration were compared. An existing groundwater flow model coupled with an advective transport simulation model of the Salt Lake Valley, Utah (USA), was adapted for calibration of tritium concentration. Two calibration approaches are used: a "complex" highly parameterized porosity field and a "simple" parsimonious model of porosity distribution. As per the results of this study, a complex model with tritium concentrations and tritium/helium apparent ages performs best. Therefore, it raises important issues related to the choice of 'optimal' simulation models which could be eventually used for optimal management strategy development.

\section{Coastal aquifer management}

Optimal management of the coastal aquifer to control and prevent saltwater intrusion in over-exploited coastal aquifers is another area where substantial efforts have been devoted to the development of optimization-based management models. Again, it is almost essential that the highly nonlinear and hydrogeologic processes, including the density-dependent advective and dispersive processes, need integration or linking of robust numerical simulation models and optimal decision models. Simplifying assumptions such as sharp interfaces between freshwater and saltwater have been made in the past to improve the computational feasibility of large-scale regional coastalaquifer-simulation models. However, incorporation of a more adequate description of the complex hydrogeologic processes requires a computationally intensive numerical simulation model linked to the optimization model. Again, the issue of computational efficiency and feasibility for multi-objective regional-scale coastal aquifer management has led to the search for approximators such as surrogate or meta models to be linked to the optimization model. In order to incorporate ubiquitous uncertainties in both modeling and predicting the physical processes, utilization of ensemble surrogate models have been proposed recently (Sreekanth and Datta 2011). Another related issue in large-scale groundwater-management-strategy development is the monitoring of compliance in accordance with prescribed strategies and dynamic correction of strategies based on field monitoring. Therefore, monitoring network design for impact assessment and compliance verification is now being integrated within the overall optimizationbased approach for coastal aquifer management. All 
these aspects are covered in coastal-aquifermanagement-related papers included in this theme issue.

Ketabchi and Ataie-Ashtiani, while reviewing the development of management models for coastal aquifers, emphasize some of the challenges and limitations to practical applications. This study summarizes the scientific basis of coastal groundwater management to discuss the present state of the art, and to outline ideas for improving computational efficiency. Included in the discussion are development of possible robust tools such as efficient evolutionary algorithms (EAs), surrogate models, and parallel processing techniques. The discussion also includes challenges related to advances in simulating and optimizing phases such as introducing new robust algorithms, implementing novel and high-performance tools, considering global concerns (e.g., climate change impacts), and enhancing the existing models to fit the complexities of real-world applications. An application to a site in Iran demonstrates that the required computational time is considerably reduced by as much as three orders of magnitude when correct combinations of robust EAs, surrogate models, and parallelization techniques are utilized.

Sreekanth and Datta emphasize the issues related to development of computationally efficient linked simulation optimization models. Both adaptive and modular genetic programming (GP) based surrogate models are discussed. These surrogate models have been successfully used as an approximate simulator of density-dependent three-dimensional (3D) advective-dispersive processes in coastal aquifers affected by saltwater intrusion due to over-exploitation. The potential for using ensemble GP models, essentially serving the role of ensemble surrogate models, is discussed and its potential relevance to reliability of estimates is mentioned. Ensemble surrogate models could be utilized to incorporate the uncertainties in parameter estimates as well as in simulation modeling. The most important aspect highlighted in this review is the practical need for continued monitoring of the prescribed management strategy implementation in terms of compliance. The other issue highlighted is the temporal deviations between the expected outcomes and actual impacts of an implemented management strategy that need to be monitored as per an adequate and optimal monitoring network design. Any management policy needs proper implementation and therefore requires compliance monitoring. Appropriate design of the monitoring scheme needs an additional mechanism of feeding back compliance information and information on deviations between planned and actual impacts of a management strategy. Hence, a sequential design and feedback-based methodology consisting of a timevarying monitoring network is a logical option.

Kourakos and Mantoglou emphasize the achievement of substantial computational efficiency in terms of drastically reducing computational time. Often the computational time required may affect the feasibility of solving a linked simulation optimization model for developing regional optimal management strategies in a coastal aquifer. A computationally efficient approach is presented which can result in substantial savings in solution time required by a linked simulation optimization model. The computational time reduction is achieved by utilizing an efficient coupling between simulation models and optimization algorithms for management of coastal aquifers. The simulation models are based on the sharpinterface approximation where the decision variables do not affect the discretized system matrix. The major procedure involved is the transformation of the system matrix prior to optimization, which supports rapid solution of the linear system during the optimization stage, for different values of decision variables. The method is applied to a coastal aquifer on the Greek island of Santorini where the proposed simulation-optimization coupling method is employed to maximize pumping rates subject to environmental constrains that protect the aquifer from seawater intrusion. Various packages were tested in order to investigate their efficiency in solving the linear system pertinent to the case study. The proposed method based on coupling of equations, is found to be very efficient in terms of reducing computational cost.

Karatzas and Dokou present an application of a saltwater-intrusion-management methodology to a coastal aquifer site in Greece. A particle-swarm-optimization algorithm is shown to be computationally efficient in solving the linked simulation-optimization model. The proposed methodology involves a weighted multiobjective optimization methodology using the particle swarm optimization algorithm combined with groundwater simulation modeling. The sharp-interface approximation combined with the Ghyben-Herztberg equation is used to estimate the saltwater-intrusion front location. The management goal is to maximize groundwater withdrawal rates in the existing pumping wells while inhibiting the saltwater-intrusion front at locations closer to the coastal zone. This is achieved by requiring a minimum hydraulichead value at pre-selected observation locations. The coastal aquifer of Malia, located in the northern part of Crete, Greece, is studied in order to formulate alternative management plans for the mitigation of the phenomenon. Two different uses, drinking-water supply and irrigation supply, were considered, while the possibility of using other sources of freshwater to supplement supply is also considered.

Park and Shi developed a simulation-optimization model to identify optimal solutions for managing both types of groundwater in coastal areas. The developed model has the potential to be used for conventional management problems of fresh groundwater development and of seawater-intrusion control. In addition, the model can be used for problems of concurrent development of fresh and saline/brackish groundwater for beneficial uses. A set of hypothetical examples demonstrate the applicability of the proposed model. In the protection of an overexploiting freshwater pumping well, the saltwater pumping scheme was less efficient than the freshwater injection scheme. Although the former scheme may be more advantageous in some limited cases, the latter should be considered first as it retains more freshwater in the aquifer. The concurrent development of fresh and brackish groundwater exhibited two different sets of optimal solutions: one with a large amount of freshwater and a 
small amount of brackish water with high salinity, and the other with a small amount of freshwater and a large amount of brackish water with low salinity.

\section{General groundwater management}

In addition to the two major areas (saltwater intrusion and source characterization) attracting the use of optimization tools for evolving optimal prescriptive solutions, the allocation, utilization and conjunctive use of groundwater and surface water has been another area that attracted the development of optimization-based management models. Also, the design of remediation strategies for contaminated aquifers requires the use of economically and physically optimal strategies. Another issue requiring further attention is the incorporation of uncertainties in parameter estimates and in the modeling of flow and transport processes. Some of these issues are addressed in following papers forming a part of this theme issue.

Medellín-Azuara et al. present a timely application, and analyze solution results, for a regional-scale optimal groundwater and surface-water-management problem for a study area located in the drought-stricken Central Valley, California (USA). In many places in California, groundwater is the major alternative water source for agriculture; therefore, groundwater availability will impact the state's water management. The allocation of surface and groundwater depends on the availability of groundwater. In this study, SWAP (an economic model of agricultural production and water use in California) and C2VSim (the California Department of Water Resources groundwater model for California's Central Valley) are connected. This paper examines the economic costs of pumping replacement groundwater during drought, and the potential loss of pumping capacity as groundwater levels drop. Results point to the potential of a portfolio approach for agriculture, in which crop mixing and conservation practices have substantial roles. This paper explores the dynamic link between surface water and groundwater use in agriculture using a case study on the 2014 California drought. The analysis shows that replacing surface water with groundwater during drought can significantly mitigate the economic cost of drought. However, additional groundwater pumping draws down the aquifer and imposes long-term costs including energy, pumping and well capital replacement costs, in addition to the standard long-term risks to water supply reliability. This paper presents an important and significant example of optimal management strategy development based on economic consideration for agricultural production.

Singh presents a review of the individual and combined applications of simulation and optimization modeling for the management of groundwater-resource problems associated with irrigated agriculture. The study revealed that the combined use of simulation-optimization modeling is very suitable for achieving an optimal solution for groundwater-resource problems, even with a large number of variables. The author concludes that independent model tools are suitable for solving the problems of uncertainty analysis and parameter estimation in groundwater modeling studies. Artificial neural networks were used to minimize the problem of computational complexity. It was also concluded that the incorporation of socioeconomic aspects into the groundwater management modeling would be an important development in future studies.

Cody et al. present a computationally efficient methodology for identifying optimal injection strategies at candidate geological carbon sequestration (GCS) sites having uncertainty associated with caprock permeability, effective compressibility, and aquifer permeability. A multi-objective evolutionary optimization algorithm is used to heuristically determine nondominated solutions for the following two conflicting objectives: (1) maximize mass of $\mathrm{CO}_{2}$ sequestered and (2) minimize project cost. A semi-analytical algorithm is used to estimate $\mathrm{CO}_{2}$ leakage mass rather than a numerical model, enabling the study of GCS sites having vastly different domain characteristics. The stochastic optimization framework is applied to a feasibility study of GCS in a brine aquifer in the Michigan Basin (MB), USA. Eight optimization test cases are performed to investigate the impact of decisionmaker (DM) preferences on Pareto-optimal objective-function values and carbon-injection strategies. This analysis shows that the feasibility of GCS at the MB test site is highly dependent upon the DM's risk-adversity preference and degree of uncertainty associated with caprock integrity. Gains in computational efficiency achieved using parallel processing and archiving are discussed.

Hernandez and Uddameri present a combined simulation-optimization model to minimize the freshwater footprint at multi-wall hydraulic fracturing sites. The model seeks to reduce freshwater use by blending it with brackish groundwater and recovered water. Time-varying water quality and quantity mass-balance expressions and drawdown calculations using the Theis solution along with the superposition principle were embedded into the optimization model and solved using genetic algorithm. The model was parameterized for representative conditions in the Permian Basin oil and gas play region with the Dockum Formation serving as the brackish water source (Texas, USA). The concentration requirements of sulfate and magnesium limited blending. The evaporation in the frac pit constrained the amount blended during summer, while well yield of the brackish (Dockum) aquifer constrained the blending during winter. The Edwards-Trinity aquifer provided the best quality water compared to the Ogallala and Pecos Valley aquifers. The developed simulation-optimization modeling framework is flexible and easily adapted for water management at other fracturing sites.

Siade et al. present the development and application of a numerical model of groundwater flow and land subsidence in order to better understand the uncertainty associated with natural recharge and to provide a tool for groundwater management. The transient model was calibrated using PEST with water-level and subsidence data; prior information was incorporated through the use of Tikhonov regularization. The effect of parameter uncertainty on the estimation of natural 
recharge was addressed using the Null-Space Monte Carlo method. A Pareto trade-off method was used to portray the reasonableness of larger natural recharge rates. The justification of the value and the effect of uncertain pumpage rates were also evaluated.

\section{Conclusion}

The collection of papers in this theme issue illustrate the broad applicability of mathematical optimization to hydrogeology. Yet the specific topics covered here offer only a glimpse of the recent developments related to the application of optimization tools to various aspects of groundwater quality and quantity management. Optimization tools and principles significantly contributed to the development of prescriptive models for optimal management not only of small scale but more importantly of large-scale water resources systems, incorporating ubiquitous uncertainties in the prediction of natural processes and the economic impacts. Application of optimization methods have also made it feasible to provide better sustainable solutions to regional-scale long-term groundwater quality and quantity management problems that would have been very difficult to obtain with other types of analysis. Adoption of optimization models and algorithms has also lead to improved hydrogeologic and geochemical parameter estimation procedures. Last but not least, although not adequately covered in this theme issue, optimization methods have found broad applicability in economic management of groundwater systems.

More importantly, this issue points out that the hydrogeology community has not only embraced the existing optimization methods, but has also contributed to advance the knowledge of the field. Examples of some of the most complex applications of optimization tools and models in this field are evident from this theme issue. Given the trends of computing power and software developments, and the need for large-scale comprehensive management of natural resources, the hydrogeologist and decision makers will increasingly continue to adopt and rely on optimization tools and optimal decision models.

Acknowledgements This thematic issue of Hydrogeology Journal was created by three guest editors: Bithin Datta and George Kourakos (the authors of this Preface), and Brian Wagner (US Geological Survey, Menlo Park, California, USA). Brian Wagner initiated the issue's theme and direction, and initially coordinated the editorial process. The overall guidance, and continuous help and cooperation of the journal's executive editor Cliff Voss, and also help and cooperation of the journal editorial office (Sue Duncan and Susanne Schemann), are gratefully acknowledged.

\section{References}

Aral M (2011) Closure on groundwater quantity and quality management. In: Groundwater quantity and quality management. American Society of Civil Engineers, Washington, DC, pp 560567. doi: 10.1061/9780784411766.ch14
Bhattacharjya R, Datta B (2005) Optimal management of coastal aquifers using linked simulation optimization approach. Water Resour Manag 19:295-320

Das A, Datta B (1999) Development of multiobjective management models for coastal aquifers. J Water Resour Plann Manag 125(2):76-87

Datta B, Peralta RC (1986) Optimal modification of regional potentiometric surface design for groundwater contaminant containment. Trans Am Soc Agric Eng 29(4):1611-1623

Datta B, Beegle JE, Kavvas ML, Orlob GT (1989) Development of an expert system embedding pattern recognition techniques for pollution source identification. Dept. of Civil and Environmental Engineering, University of California, Davis, CA, Project completion report submitted to USGS, National Technical Information Service, Alexandria, VA

Datta B, Chakrabarty D, Dhar A (2009) Simultaneous identification of unknown groundwater pollution sources and estimation of aquifer parameters. J Hydrol 376(1, 2):48-57

Datta B, Chakrabarty D, Dhar A (2011) Identification of unknown groundwater pollution sources using classical optimization with linked simulation. J Hydro Environ Res 5(1):25-36

Dhar A, Datta B (2009) Saltwater intrusion management of coastal aquifers, I: linked simulation-optimization. J Hydrol Eng 14(12):1263-1272

Doherty JE, Hunt RJ (2010) Approaches to highly parameterized inversion: a guide to using PEST for groundwatermodel calibration. US Geol Surv Sci Invest Rep 2010-5169, $59 \mathrm{pp}$

Gorelick SM (1983) A review of distributed parameter groundwater management modeling methods. Water Resour Res 19(2):305-319

Gorelick SM, Remson I (1982) Optimal dynamic management of groundwater pollutant sources. Water Resour Res 18(1):71-76. doi:10.1029/WR018i001p00071

Gorelick SM, Evans B, Remson I (1983) Identifying sources of groundwater pollution: an optimization approach. Water Resour Res 19(3):779-790

Gorelick SM, Voss CI, Gill PE, Murray W, Saunders MA, Wright MH (1984) Aquifer reclamation design: the use of contaminant transport simulation combined with nonlinear programming. Water Resour Res 20(4):415-427. doi:10.1029/ WR020i004p00415

Illangasekare T, Morel-Seytoux HJ (1982) Stream-aquifer influence coefficients as tools for simulation and management. Water Resour Res 18(1):168-176

Kourakos G, Mantoglou A (2009) Pumping optimization of coastal aquifers based on evolutionary algorithms and surrogate modular neural network models. Adv Water Res 32(4):507521. 10.1016/j.advwatres.2009.01.001

McPhee J, Yeh WWG (2004) Multi-objective optimization for sustainable groundwater management in semi arid regions. Water Resour Plan Manag ASCE 130(6):490-497

Mirghani BY, Mahinthakumar KG, Tryby ME, Ranjithan RS, Zechman EM (2009) A parallel evolutionary strategy based simulation-optimization approach for solving groundwater source identification problems. Adv Water Resour 32:1373-1385

Sreekanth J, Datta B (2011) Coupled simulation-optimization model for coastal aquifer management using genetic programmingbased ensemble surrogate models and multiple-realization optimization. Water Resour Res 47(4). doi:10.1029/ 2010WR009683

Wagner BJ (1995) Recent advances in simulation-optimization groundwater management modeling. Rev Geophys 33(S2):1021-1028. doi:10.1029/95RG00394

Wagner BJ, Gorelick SM (1989) Reliable aquifer remediation in the presence of spatially variable hydraulic conductivity: from data to design. Water Resour Res 25(10):2211-2225. doi:10.1029/ WR025i010p02211

Yeh WW-G (1986) Review of parameter identification procedures in groundwater hydrology: the inverse problem. Water Resour Res 22(2):95-108 